\title{
The Number of Labeled 2-Connected Planar Graphs
}

\author{
Edward A. Bender \\ Department of Mathematics \\ University of California at San Diego \\ La Jolla, CA 92093-0112, USA \\ ebender@ucsd.edu \\ Zhicheng Gao* \\ School of Mathematics and Statistics \\ Carleton University \\ Ottawa K1S 5B6, Canada \\ zgao@math. carleton.ca \\ Nicholas C. Wormald ${ }^{\dagger}$ \\ Department of Mathematics and Statistics \\ University of Melbourne \\ VIC 3010, Australia \\ nick@ms.unimelb.edu.au
}

Submitted: April 9, 2001; Revised November 3, 2002; Accepted: November 10, 2002.

MR Subject Classifications: 05C30, 05A16

\begin{abstract}
We derive the asymptotic expression for the number of labeled 2-connected planar graphs with respect to vertices and edges. We also show that almost all such graphs with $n$ vertices contain many copies of any fixed planar graph, and this implies that almost all such graphs have large automorphism groups.
\end{abstract}

${ }^{*}$ Research supported by the Australian Research Council and NSERC

${ }^{\dagger}$ Research supported by the Australian Research Council 


\section{Introduction}

A (planar) map is a connected graph embedded in the sphere. A planar graph is a connected graph which can be embedded in the sphere. Throughout the paper, unless stated otherwise, all planar maps and graphs have no loops or multiple edges. Since a single graph may have many embeddings, there are generally fewer planar graphs than there are maps. In this paper, we study the number of labeled 2-connected planar graphs with a given number of vertices and edges.

Symmetry causes difficulties in the enumeration of both graphs and maps. In graphical enumeration, one destroys symmetry by labeling the vertices. In map enumeration, it is simpler to destroy symmetry by a Tutte rooting: select an edge, a direction on the edge, and a side of the edge.

In enumerating $c$-connected graphs or maps, it is natural to proceed from 1connected to 2-connected and thence to 3-connected by means of functional compositions based on theorems about graphical construction. This scheme has not yet been implemented for enumerating $c$-connected planar graphs because of the absence of any direct method of enumerating 1-connected planar graphs. However, we are able to proceed in the opposite direction by making use of known results on map enumeration, as well as the fact that a 3-connected planar graph has only one embedding in the sphere [10]. There are $n$ ! ways to label a rooted $n$-vertex map and $4 q$ ways to root a labeled map with $q$ edges which is not just a path. Hence, if $m_{c}(n, q)$ (resp. $\left.g_{c}(n, q)\right)$ is the number of $c$-connected $n$-vertex, $q$-edge rooted maps (resp. labeled planar graphs) with $n>c$, then

$$
m_{c}(n, q) n !=g_{c}(n, q)(4 q) \quad \text { for } c \geq 3
$$

since both sides count rooted, labeled $c$-connected planar maps.

We note that, when $m_{c}(n, q) \neq 0$, we have

$$
n-1 \leq q \leq 3 n-6 .
$$

The first inequality follows from connectivity. The latter follows from Euler's formula $V-E+F=2$ and the fact that the absence of loops and multiple edges guarantees that each face has at least three sides. We also note that, for 2-connected graphs with at least 3 vertices, $q \geq n$.

We use the following functions of $t$ in the rest of the paper.

$$
\begin{aligned}
D_{3}= & 384 t^{3}(1+t)^{2}(1+2 t)^{2}(3+t)^{2} \alpha^{3 / 2} \beta^{-5 / 2} \\
x_{0}= & \frac{(1+3 t)(1-t)^{3}}{16 t^{3}} \\
y_{0}= & \frac{1+2 t}{(1+3 t)(1-t)} e^{-h}-1 \\
\mu= & \frac{(1+t)(3+t)^{2}(1+2 t)^{2}(1+3 t)^{2} y_{0}}{t^{3}\left(1+y_{0}\right) \alpha} \\
\sigma^{2}= & \frac{(3+t)^{2}(1+2 t)^{2}(1+3 t)^{2} y_{0}}{3 t^{6}(1+t)\left(1+y_{0}\right)^{2} \alpha^{3}}\left(3 t^{3}(1+t)^{2} \alpha^{2}\right. \\
& \left.\quad-(1-t)(3+t)(1+2 t)(1+3 t)^{2} y_{0} \gamma\right), \quad \sigma>0 .
\end{aligned}
$$


where

$$
\begin{aligned}
\alpha= & 144+592 t+664 t^{2}+135 t^{3}+6 t^{4}-5 t^{5} \\
\beta= & 3 t(1+t)\left(400+1808 t+2527 t^{2}+1155 t^{3}+237 t^{4}+17 t^{5}\right) \\
\gamma= & 1296+10272 t+30920 t^{2}+42526 t^{3}+23135 t^{4} \\
& -1482 t^{5}-4650 t^{6}-1358 t^{7}-405 t^{8}-30 t^{9} \\
h= & \frac{t^{2}(1-t)\left(18+36 t+5 t^{2}\right)}{2(3+t)(1+2 t)(1+3 t)^{2}} .
\end{aligned}
$$

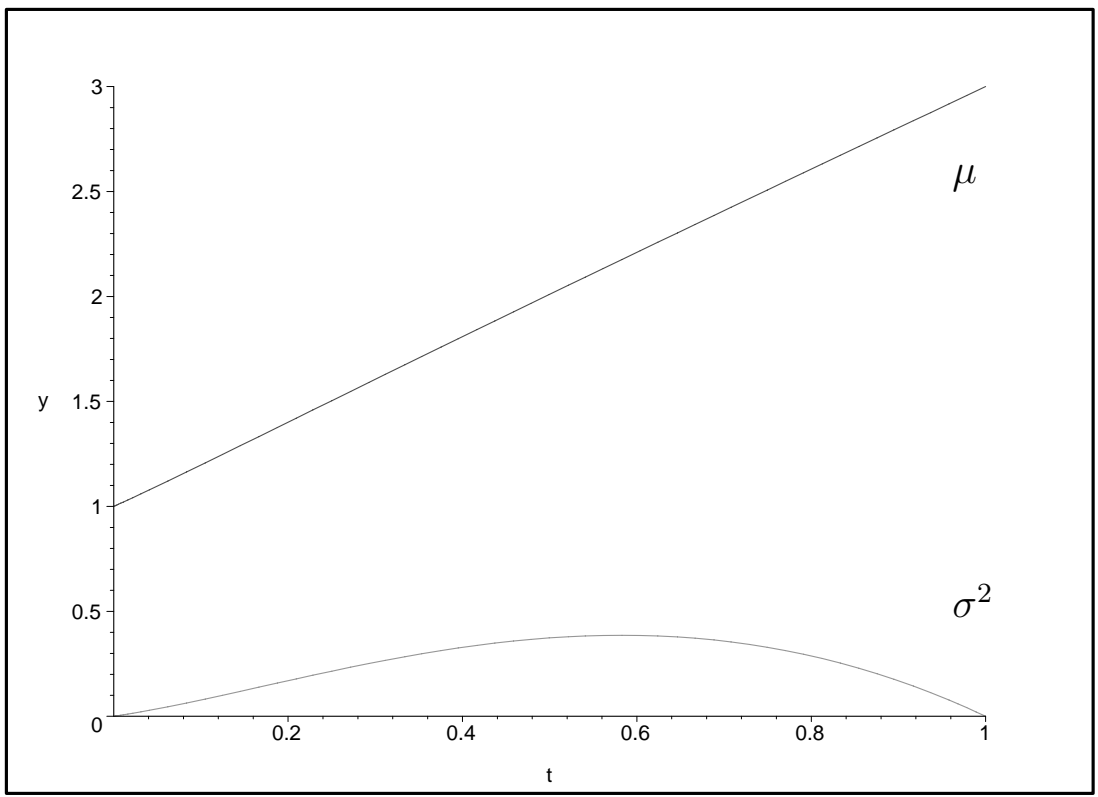

Figure 1: The plots of $\mu$ and $\sigma^{2}$ for $0 \leq t \leq 1$.

For labeled 2-connected planar graphs we have the following.

Theorem 1 Let $J$ be any closed subinterval of $(1,3)$, and $D_{3}, x_{0}, y_{0}, \mu=\mu(t), \sigma$ be as defined in (3)-(7). Then

(a) For $q_{0} / n \in J$, there is a unique $t \in(0,1)$ such that $\mu(t)=q_{0} / n$, and

$$
g_{2}(n, q)=\frac{3 x_{0}^{2} y_{0} D_{3} n !}{8 \sqrt{2} \pi\left(1+y_{0}\right) \sigma n^{3} q} x_{0}^{-n} y_{0}^{-q}\left(\exp \left\{-\frac{\left(q-q_{0}\right)^{2}}{2 n \sigma^{2}}\right\}+o(1)\right)
$$

uniformly as $n \rightarrow \infty$ and $q_{0} / n \in J$.

(b) There is a unique real root $0<t<1$ of $y_{0}(t)=1$, namely $t=t(1) \approx 0.62637$. At $t=t(1)$, we have

$$
x_{0} \approx 0.03819, \quad \mu \approx 2.2629, \quad D_{3} \approx 0.05433,
$$




$$
g_{2}(n)=\sum_{q} g_{2}(n, q) \sim \frac{3 x_{0}^{2} D_{3} n !}{16 \mu \sqrt{\pi}} n^{-7 / 2} x_{0}^{-n}, \quad n \rightarrow \infty,
$$

and for fixed $n$, the maximum value of $g_{2}(n, q)$ is achieved at $q=\mu n+$ $o\left(n^{1 / 2}\right) \approx 2.2629 n$.

In view of $(2)$, the constraint that $q_{0} / n$ lies in a closed subinterval of $(1,3)$ is not too severe. Figures 1 and 2 show the plots of $\mu, \sigma^{2}$ and $\mu^{\prime}(t)$ for $0 \leq t \leq 1$.

We will also prove the following subgraph density result which is similar to the submap density result proved in [2]. Let $G$ be a planar graph. A copy of a planar graph $G_{0}$ in $G$ means a subgraph (not necessarily induced) of $G$ which is isomorphic to $G_{0}$. A network is a planar graph with two special vertices, called poles, such that adding the edge between the poles creates a 2-connected planar graph. A copy of a network $G_{1}^{+}$in $G$ is a subgraph of $G$ which is isomorphic to $G_{1}^{+}$and whose non-polar vertices are incident with no edges in $E(G) \backslash E\left(G_{1}^{+}\right)$.

Theorem 2 For any fixed network $G_{1}^{+}$, there exist positive constants $c$ and $\delta$ such that the probability that a random labeled 2-connected planar graph $G$ with $n$ vertices has less than cn vertex disjoint copies of $G_{1}^{+}$is $O\left(e^{-\delta n}\right)$.

We immediately obtain from this the desired result for subgraphs, because any fixed planar graph is a subgraph of some network minus its poles.

Corollary 1 For any fixed planar graph $G_{0}$, there exist positive constants $c$ and $\delta$ such that the probability that a random labeled 2-connected planar graph $G$ with $n$ vertices has less than cn vertex disjoint copies of $G_{0}$ is $O\left(e^{-\delta n}\right)$.

It is interesting to note that almost all graphs or maps have no symmetries. (See [11] for graphs; see [7] and [1] for maps.) The situation is different for 2connected planar graphs:

Theorem 3 There is a constant $C>1$ such that almost all 2-connected planar graphs $G$ (in the sense of labeled or unlabeled counting) have an automorphism group of order at least $C^{v(G)}$, where $v(G)$ is the number of vertices of $G$.

As can be seen from the proof (given later) this result can be extended to many other classes of planar graphs.

Let $M_{c}(x, y)=\sum_{n, q} m_{c}(n, q) x^{n} y^{q}$ and $G_{c}(x, y)=\sum_{n, q} g_{c}(n, q) x^{n} y^{q} / n !$. If one wants to allow multiple edges in 2-connected planar graphs, then the generating function is $G_{2}\left(x, \frac{y}{1-y}\right)$. If one wants to allow loops and multiple edges in 1-connected planar graphs, the generating function is $G_{1}\left(\frac{x}{1-y}, \frac{y}{1-y}\right)$. We do not pursue these possibilities. It would be of great interest to obtain similar results for all connected planar graphs, but this appears to be more difficult.

We used Maple to assist us with the algebraic manipulations in this paper. 


\section{The Functional Equation for 2-connected Pla- nar Graphs}

Before studying $G_{2}$ we need some information about $M_{3}$. It follows from (1) that

$$
M_{c}(x, y)=\frac{\partial G_{c}(x, y)}{\partial y} 4 y \quad \text { for } c \geq 3 .
$$

Mullin and Schellenberg [5] obtained a generating function $Q_{N}^{*}(X, Y)$ in which the coefficient of $X^{n-1} Y^{m-1}$ counts rooted 3 -connected $n$-vertex $m$-face maps. Using Euler's relation we have $M_{3}(x, y)=x Q_{N}^{*}(x y, y)$ and so, from [5],

$$
M_{3}(x, z)=x^{2} z^{2}\left(\frac{1}{1+x z}+\frac{1}{1+z}-1-\frac{(1+u)^{2}(1+v)^{2}}{(1+u+v)^{3}}\right)
$$

where

$$
u=x z(1+v)^{2} \quad \text { and } \quad v=z(1+u)^{2}
$$

determine $u$ and $v$ implicitly as power series in $x$ and $y$ with nonnegative coefficients.

The next lemma uses a result of Walsh to relate $G_{2}$ to $M_{3}$.

Lemma 1 We have

$$
\frac{\partial G_{2}(x, y)}{\partial y}=\frac{x^{2}}{2}\left(\frac{1+D}{1+y}-1\right)
$$

where the power series $D$ is defined implicitly by $D(x, 0)=0$ and

$$
\frac{M_{3}(x, D)}{2 x^{2} D}-\log \left(\frac{1+D}{1+y}\right)+\frac{x D^{2}}{1+x D}=0
$$

The coefficients of $D(x, y)$ are nonnegative.

Proof: Walsh [9] provides a functional equation relating the generating functions for the numbers of graphs in two classes, such that the first class is a set of 3connected graphs and the second consists of all the 2-connected graphs whose 3connected "components" are in the first class. The discussion by Tutte [8], with an application to counting 3-connected rooted maps, is helpful to understand the definition of a 3-connected component (called a 3-connected core by Tutte). The following is a brief description which is adapted to defining the components rather than counting. Given a 2-vertex cut $\{u, v\}$ of a 2-connected graph $G$, and a component $C$ of $G-\{u, v\}$, define the graph $G(C)$ as the subgraph of $G$ induced by $V(C) \cup\{u, v\}$, together with the edge $u v$ if not already there. One may reduce a 2-connected graph $G$ to its "components" by replacing $G$ by the graphs $G(C)$ at one of its 2-vertex cuts, and then recursively applying this operation to any graph which results. The 3-connected graphs which finally result from this are the 3-connected "components" of $G$. (It is not hard to verify from either Walsh's or Tutte's presentations that the only other graphs finally resulting are triangles, which result from slicing up the "polygons" of Tutte; the "bonds" of Tutte are simply dismantled in this process. Tutte's polygons and bonds correspond respectively to the s-networks and p-networks of Walsh.) 
It is clear that the set of graphs whose 3-connected "components" are planar is precisely the set of planar 2-connected graphs. So by [9, Proposition 1.2 and equations (8)-(11)] applied to $G_{2}$ and $G_{3}$,

$$
\begin{aligned}
\frac{2 \partial G_{3}(x, D)}{x^{2} \partial D} & =\log (K(x, y))-P(x, y) \\
K(x, y) & =\frac{2}{x^{2}} \frac{\partial\left(G_{2}(x, y)+x^{2} y / 2\right)}{\partial y} \\
D(x, y) & =(1+y) K(x, y)-1 \\
P(x, y) & =x D(x, y)(D(x, y)-P(x, y)) .
\end{aligned}
$$

Since the last two equations are easily solved for $K$ and $P$, the second equation becomes (11) and the first becomes (12) when (8) is used. Since $G_{2}$ has nonnegative

coefficients, so does $\frac{1+D}{1+y}$ and hence $1+D$ as well. Since $D$ has no constant term, we are done.

\section{Proof of Theorem 1}

The proof of Theorem 1 has three main steps:

(A) Determine the dominant singularities of the function $D(x, y)$ in Lemma 1 , when it is viewed as a function of $x$ with $y$ fixed.

(B) Find the asymptotic expansion of $D(x, y)$ at the dominant singularities.

(C) Apply a local limit theorem to obtain the asymptotics of $\left[x^{n} y^{q}\right] D(x, y)$.

Throughout this section, any claim involving $\epsilon$ carries the implicit assumption that $\epsilon>0$ and that the claim holds for $\epsilon$ sufficiently small. We use $I$ to denote any closed subinterval of $(0, \infty)$, and $T$ to denote any closed subinterval of $(0,1)$. We also define

$$
I_{\epsilon}=\{z:|z| \in I,|\operatorname{Arg}(z)| \leq \epsilon\},
$$

and define $T_{\epsilon}$ similarly.

We first prove the following technical lemma which is needed to study the behavior of the singularities of $D(x, y)$. It also establishes the uniqueness of $t(1)$ that was claimed in Theorem 2 .

Lemma 2 Let $y_{0}=y_{0}(t)$ be as defined in (5). Then $y_{0}(t)$ has an analytic inverse function for $t \in T_{\epsilon}$, and $y_{0}(t)$ increases from 0 to $\infty$ as $t$ increases from 0 to 1 .

Proof: Note that

$$
y_{0}^{\prime}(t)=\frac{3 t^{2}(1+t) \alpha}{(1-t)^{2}(1+3 t)^{4}(1+2 t)(3+t)^{2}} e^{-h}>0 \quad \text { for } 0<t<1 .
$$

Hence $y_{0}^{\prime}(t)$ is never zero in $T_{\epsilon}$, and it is a 1-1 mapping for $t \in T_{\epsilon}$. Therefore equation (5) defines a function $t\left(y_{0}\right)$ which is analytic and 1-1 in $I_{\epsilon}$. It is clear that $y_{0} \rightarrow \infty$ as $t \rightarrow 1-$, and $y_{0} \rightarrow 0+$ as $t \rightarrow 0+$. 
Lemma 3 Fix $y_{0} \in I_{\epsilon}$. Let $t=t\left(y_{0}\right)$ be the inverse function in Lemma 2 and let $x_{0}=x_{0}(t)$ be given by (4).

(i) $D\left(x, y_{0}\right)$ has a unique singularity on its circle of convergence and the singularity is given by $x_{0}$.

(ii) Fix $\varphi$ with $0<\varphi<\pi / 2$. For sufficiently small $\delta, D\left(x, y_{0}\right)$ is analytic in the region

$$
\Delta\left(y_{0}, \delta\right)=\left\{z:|z| \leq(1+\delta)\left|x_{0}\right|,\left|\operatorname{Arg}\left(z / x_{0}-1\right)\right| \geq \varphi, z \neq x_{0}\right\} .
$$

(iii) For each fixed $y \neq 0$ let $r(y)$ be the radius of convergence of $D(x, y)$. Then $r(y) \geq r(|y|)$ with equality if and only if $y$ is a positive real.

Proof: Since $D(x, y)$ is defined by (12), there are three possible sources for the singularities:

(a) the singularities of $M_{3}$,

(b) a branch point in solving (12), and

(c) $1+x D=0$ and/or $\log ((1+D) /(1+y))$ becomes unbounded.

We first deal with positive $y_{0}$ (i.e. $0<t<1$ ), the general statement for $y_{0} \in I_{\epsilon}$ then follows from continuity. For each positive $z$, the singularities of $M_{3}(x, z)$ were studied in [4], and it was shown that the singularity $x_{0}$ is related to $z$ by equations (10) and the equation $1+u+v-3 u v=0$ with $x=x_{0}$. Setting

$$
u=\frac{1}{3 t}
$$

in the latter equation, we obtain

$$
v=\frac{t+3}{3(t-1)}
$$

and $x_{0}$ as given in Section 1. Replacing $z$ by $D$ and using equations (12) and (9), we obtain the formula for $y_{0}(t)$ in Section 1 and

$$
D_{0}=D\left(x_{0}, y_{0}\right)=\frac{3 t^{2}}{(1-t)(1+3 t)} .
$$

To show that $x_{0}$ is the unique singularity on the circle of convergence of $D\left(x, y_{0}\right)$, we need to show that sources (b) and (c) do not provide singularities in the disk $|x| \leq x_{0}$.

We first consider source (b). If the left side of (12) is called $H(D, y)$, then

$$
H_{y}=\frac{\partial H}{\partial y}=\frac{1}{1+y}
$$

and

$$
H_{D}=\frac{\partial H}{\partial D}=\frac{\partial\left\{M_{3}(x, D) / D\right\}}{2 x^{2} \partial D}-\frac{1-x D^{2}(2+x D)}{(1+D)(1+x D)^{2}}
$$


Since $x_{0}>0, D_{0}=D\left(x_{0}, y_{0}\right)>0$ and the power series for $D$ and $M_{3}$ has nonnegative coefficients, we have

$$
\begin{aligned}
\left|H_{D}(x, D)\right| & \geq\left|\frac{1-x D^{2}(2+x D)}{(1+D)(1+x D)^{2}}\right|-\left|\frac{\partial\left\{M_{3}(x, D) / D\right\}}{2 x^{2} \partial D}\right| \\
& \geq \frac{1-x_{0} D_{0}^{2}\left(2+x_{0} D_{0}\right)}{\left(1+D_{0}\right)\left(1+x_{0} D_{0}\right)^{2}}-\left.\frac{\partial\left\{M_{3}(x, D) / D\right\}}{2 x^{2} \partial D}\right|_{x=x_{0}, D=D_{0}} \\
& =\frac{t^{2}(1-t)\left(400+1808 t+2527 t^{2}+1155 t^{3}+237 t^{4}+17 t^{5}\right)}{2(1+3 t)^{2}(1+2 t)^{2}(3+t)^{2}}
\end{aligned}
$$

where the last expression is obtained by using (9), (10) and Maple. Hence $\left|H_{D}(x, D)\right|>$ 0 when $|x| \leq x_{0}$, and therefore $x$ is not a singularity from source (b).

Next we consider source (c). Since $M_{3}(x, D)$ is well defined, it follows from (12) that the last two terms must both be unbounded. Hence $1+x D=0$ and $1+D=0$. So $x=1$ and $D=-1$, which contradicts the fact that $D\left(1, y_{0}\right)>0$. Since $y_{0}$ is in a very small neighborhood of a compact set, claims (i) and (ii) follow from continuity.

To prove (iii), we first note that the singularities from source (a) satisfy (iii) by [4]. Hence we only need to consider singularities arising from sources (b) and (c). Since $D(x, y)$ has nonnegative coefficients, we have $r(y) \geq r(|y|)$. Suppose $x=x(y)$ is a singularity from source (b) satisfying $|x(y)|=r(|y|)$ for some $y \neq|y|$. Then inequality (16) would lead to the same contradiction. Now suppose $x=x(y)$ is a singularity from source (c) satisfying $|x(y)|=r(|y|)$ for some $y \neq|y|$. As shown above, it follows that $x(y)=1$ and $D(x, y)=-1$. Since $r(|y|)=|x(y)|=1$, using Lemma 2 we obtain $|y| \approx 0.1879$ and the corresponding value of $t$ is $t=1 / 3$. Hence $D(1,|y|)=1 / 4$, which contradicts $1=|D(1, y)| \leq D(1,|y|)$.

Now we carry out step (B). Replace $z$ by $D$ in (9) and (10). Let $y$ and $t$ be related as in Lemma 2 and fix $y$. The four equations (9), (10), and (12) contain the five variables $x, u, v, M_{3}$, and $D$. Using (9) and the second equation in (10), we can simply eliminate $M_{3}$ and $v$ to obtain two equations in $x, u$ and $D$. From these two equations we can see that $u$ and $D$ have asymptotic expansions in $X=\sqrt{1-x / x_{0}}$ around the singularity $x_{0}$. Substituting $D=\sum D_{k} X^{k}$ and $u=\sum u_{k} X^{k}$ into these two equations, and equating coefficients of powers of $X$, we obtain

$D_{0}=D\left(x_{0}, y_{0}\right)=\frac{3 t^{2}}{(1-t)(1+3 t)}, \quad D_{1}=0, D_{2}=-\frac{48 t(1+t)(1+2 t)^{2}\left(18+6 t+t^{2}\right)}{(1+3 t) \beta}$,

and (3). Using (2) and the "transfer theorem" [6, Theorem 11.4], we obtain

$$
\left[x^{n}\right] \frac{\partial G_{2}(x, y)}{\partial y} \sim \frac{x_{0}^{2} D_{3}}{2(1+y) \Gamma(-3 / 2)} n^{-5 / 2} x_{0}^{-n},
$$

uniformly for all $t \in T_{\epsilon}$.

Setting $y_{0}=1$, i.e. $t=t(1) \approx 0.62637$, and applying [3, Theorem 1$]$, we see that the sequence $\left\{q g_{2}(n, q) / g_{2}(n)\right\}$ is asymptotically normal with mean $q_{0}=\mu n$ and variance $n \sigma^{2}$ given by (6) and (7) evaluated at $y_{0}=1$. It follows that the number of edges is sharply concentrated around $q_{0}$, and hence the asymptotics for $g_{2}(n)$ as 
stated in Theorem 1(b) follows. Theorem 1(a) follows from Lemma 3, (17), and [3, Theorem 2]. The shifted mean and variance are calculated using the formulas

$$
\frac{q_{0}}{n}=\mu=-\frac{y_{0}}{x_{0}} \frac{d x_{0}}{d y_{0}}=-\frac{y_{0}}{x_{0} y_{0}^{\prime}(t)} \frac{d x_{0}}{d t} \text { and } \sigma^{2}=y_{0} \frac{d \mu}{d y_{0}}=\frac{y_{0}}{y_{0}^{\prime}(t)} \frac{d \mu}{d t},
$$

which are functions of $t$ as given in (5) and (6). Using Maple, we find that $\mu(0)=1$, $\mu(1)=3$, and $\mu^{\prime}(t)>0$ is between 1.88 and 2.05 for $0 \leq t \leq 1$. (See Figures 1 and 2 ). Hence $q_{0} / n$ increases from 1 to 3 as $t$ increases from 0 to 1 . This finishes the proof of Theorem 1.

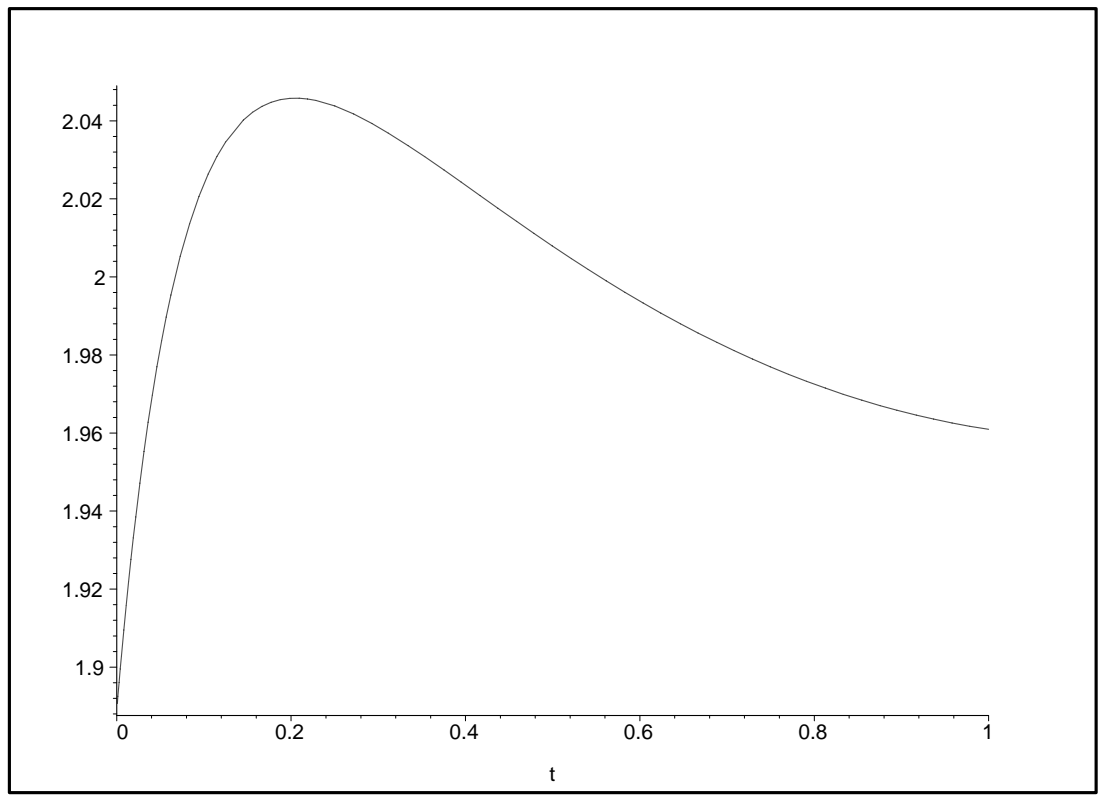

Figure 2: The plot of $\mu^{\prime}(t)$ for $0 \leq t \leq 1$.

\section{Proof of Theorems 2 and 3}

Proof of Theorem 2 : One can use the same type of arguments as those in [2] and the reader may wish to look at that paper for details. First, it is easy to see that $G_{1}^{+}$can be embedded in a larger network $G_{2}^{+}$such that any two copies of $G_{2}^{+}$ in a 2-connected planar graph must be vertex disjoint except perhaps at the poles, and also such that the vertices of $G_{1}^{+}$do not contain the poles of $G_{2}^{+}$. We will prove theorem with $G_{1}^{+}$replaced by $G_{2}^{+}$and with 'vertex disjoint' replaced by 'edge disjoint'. Since edge disjoint copies of $G_{2}^{+}$contain vertex disjoint copies of $G_{1}^{+}$, the theorem will then follow. Note that all copies of $G_{2}^{+}$must be edge disjoint. 
Let $u_{1}$ and $u_{2}$ be the poles of $G_{2}^{+}$, whose other vertices are labelled. Let $G(x)$ be the exponential generating function, by number of vertices, for the number of labeled 2-connected planar graphs with less than $c$ copies of $G_{2}^{+}$, where $c$ will be chosen sufficiently small later in the proof. Now insert some copies of $G_{2}^{+}$into the 2connected planar graphs counted by $G(x)$ by selecting a graph $G$, selecting a subset of the edges of $G$ and, for each edge $v_{1} v_{2}$ selected, identifying $u_{i}$ with $v_{i}$ for $i=1$ and 2. After insertion, the whole graph is relabelled using the labels $\{1, \ldots, n\}$ (where $n$ is the number of vertices in the final graph) but retaining the ordering of the labels within each copy of $G_{2}^{+}$and on the vertices of $G$. The resulting graph, $H$, is clearly 2connected and planar. Keep the inserted copies of $G_{2}^{+}$distinguished from any others that were already present in $G$, and denote the exponential generating function counting such labelled graphs by $H(x)$. Equivalently, $H(x)$ counts the multiset of all graphs which result from the above operation applied in every possible way. Suppose $G_{2}^{+}$has $k$ vertices other than the poles. Since the number of vertices in a connected graph never exceeds the number of edges by more than 1 , the coefficients of

$$
\frac{x G\left(x+x^{k} / k !\right)}{x+x^{k} / k !}
$$

provides a lower bound on the coefficients of $H(x)$. Thus by Lemma 2 of [2], the radii of convergence, $r_{G}$ and $r_{H}$, of $G(x)$ and $H(x)$ respectively satisfy

$$
r_{G} \geq r_{H}+r_{H}^{k} / k !
$$

Let $A(x)$ denote $\sum_{n \geq 0} g_{2}(n) x^{n} / n$ !, i.e. the exponential generating function for all 2connected planar graphs counted by vertices, and let $r_{A}$ be its radius of convergence. If the multiset counted by $H(x)$ contains at most $B_{1} n B^{n}$ copies of each graph (for positive constants $B_{1}$ and $B$ ), then $r_{A} \leq B r_{H}$. If $B$ is sufficiently near 1 , it follows from (18) that $r_{G}>r_{A}$. The result now follows from the fact that Theorem 1 shows smoothness of the coefficients of $A(x)$, i.e. $\liminf _{n \rightarrow \infty}\left(g_{2}(n) / n !\right)^{1 / n}=1 / r_{A}$. It remains to show that $B$ can be made arbitrarily close to 1 . The overcount in having nonoverlapping distinguished copies of $G_{2}^{+}$in $H$ can be estimated by choosing the at most $c n$ copies of $G_{2}^{+}$which are not distinguished, in at most

$$
\sum_{i \leq c n}\left(\begin{array}{c}
n \\
i
\end{array}\right) \leq c n\left(\frac{n}{c n / e}\right)^{c n}=c n(e / c)^{c n}
$$

ways. So for $c$ sufficiently small, $B$ is sufficiently near 1 .

Proof of Theorem 3 : Let $a(G)$ be the number of automorphisms of an unlabeled $n$-vertex graph $G$. The number of distinct labelings of $G$ is $n ! / a(G)$. If $f(\cdot)$ is a statistic on graphs, its expectation on labeled graphs is

$$
\mathbf{E}_{L}(f)=\frac{\sum_{L} f(G)}{\sum_{L} 1}
$$

where the sum is over labeled graphs. Its expectation on unlabeled graphs is

$$
\mathbf{E}_{U}(f)=\frac{\sum_{U} f(G)}{\sum_{U} 1}=\frac{\sum_{L} f(G)(a(G) / n !)}{\sum_{L} a(G) / n !}=\frac{\mathbf{E}_{L}(f a)}{\mathbf{E}_{L}(a)}
$$


Let $f(G)=1$ or 0 according as the automorphism group of $G$ has order at least $C^{n}$ or not. Then it is not difficult to see that

$$
\mathbf{E}_{L}(f a) \geq \mathbf{E}_{L}(f) \mathbf{E}_{L}(a)
$$

and hence

$$
\mathbf{E}_{U}(f) \geq \mathbf{E}_{L}(f) .
$$

Therefore, the probability that the number of automorphisms of a graph with $n$ vertices exceeds $C^{n}$ is non-decreasing as we move from labeled to unlabeled graphs. Thus it suffices to prove Theorem 3 for the labeled case. We only need to choose a network $G_{1}^{+}$which has a nontrivial automorphism fixing the poles. For example, we can choose $G_{1}^{+}$be the 4-cycle abcd with vertices $a$ and $c$ being the poles. By Theorem 2, a random labeled 2-connected planar graph $G$ with $n$ vertices almost surely contains at least $c n$ copies of $G_{1}^{+}$for some positive constant $c$, so $G$ has at least $2^{c n}$ automorphisms.

\begin{tabular}{||l||r|r|r|r|r|r|r|r||}
\hline$q \backslash n$ & 3 & 4 & 5 & 6 & 7 & 8 & 9 & 10 \\
\hline 3 & 1 & & & & & & & \\
\hline 4 & & 3 & & & & & & \\
\hline 5 & & 6 & 12 & & & & & \\
\hline 6 & & 1 & 70 & 60 & & & & \\
\hline 7 & & & 100 & 720 & 360 & & & \\
\hline 8 & & & 45 & 2445 & 7560 & 2520 & & 20160 \\
\hline 9 & & & 10 & 3525 & 46830 & 84000 & 997920 & 181440 \\
\hline 10 & & & & 2637 & 132951 & 835800 & & 12700800 \\
\hline 11 & & & & 1125 & 210861 & 3915240 & 14757120 & 264600000 \\
\hline 12 & & & & 195 & 205905 & 10549168 & 103692960 & \\
\hline 13 & & & & & 123795 & 18092368 & 423918432 & 2623622400 \\
\hline 14 & & & & & 40950 & 20545920 & 1119730032 & 15189491520 \\
\hline 15 & & & & & 5712 & 15337560 & 2014030656 & 57178432080 \\
\hline 16 & & & & & & 7193760 & 2516883516 & 148486353165 \\
\hline 17 & & & & & & 1922760 & 2181661020 & 274551234345 \\
\hline 18 & & & & & & 223440 & 1285377660 & 366723015750 \\
\hline 19 & & & & & & & 491282820 & 354648836430 \\
\hline 20 & & & & & & & 109907280 & 245949836265 \\
\hline 21 & & & & & & & 10929600 & 119267623125 \\
\hline 22 & & & & & & & & 38411074800 \\
\hline 23 & & & & & & & & 7383474000 \\
\hline 24 & & & & & & & & 641277000 \\
\hline
\end{tabular}

Table 1: The number of labeled 2-connected planar graphs with $n$ vertices and $q$ edges 


\section{Tables}

In Table 1 we give the number of labeled 2-connected planar graphs with up to 10 vertices. Equations (9), (10) and (11) are used to compute the coefficients of $D(x, y)$ recursively. The coefficients of $G_{2}(x, y)$ are then computed using (11). The computation is done with the help of Maple.

Including an intermediate step of computing $u$ and $v$ as functions of $x$ and $y$ lets Maple proceed more efficiently. By this means we produced the numbers in Table 2, which compares the asymptotic formula

$$
s(n, q)=\frac{3 x_{0}^{2} y_{0} D_{3} n !}{8 \sqrt{2} \pi\left(1+y_{0}\right) \sigma n^{3} q} x_{0}^{-n} y_{0}^{-q}
$$

obtained in Theorem 1(a) with $q=q_{0}$, to the exact numbers. The exact numbers for $n=24$ are too long to be shown here. For example, the maximum over $q$ is

71811718414061789271689855235722635267872429720 ,

for $q=50$. The approximation becomes less accurate when $q / n$ approaches the

\begin{tabular}{|c|c|c|c|c|c|}
\hline & \multicolumn{3}{|c|}{$n=12$} & \multicolumn{2}{|r|}{$n=24$} \\
\hline$q / n$ & $q$ & $g_{2}(n, q)$ & $s(n, q) / g_{2}(n, q)$ & $q$ & $s(n, q) / g_{2}(n, q)$ \\
\hline $13 / 12$ & 13 & 2514758400 & 1.020 & 26 & 0.832 \\
\hline $14 / 12$ & 14 & 93165811200 & 0.837 & 28 & 0.860 \\
\hline $15 / 12$ & 15 & 1633467306240 & 0.881 & 30 & 0.924 \\
\hline $16 / 12$ & 16 & 16678334506680 & 0.970 & 32 & 0.980 \\
\hline $17 / 12$ & 17 & 110896065403200 & 1.074 & 34 & 1.028 \\
\hline $18 / 12$ & 18 & 513156897696300 & 1.185 & 36 & 1.074 \\
\hline $19 / 12$ & 19 & 1723180985236260 & 1.305 & 38 & 1.120 \\
\hline $20 / 12$ & 20 & 4315117359458136 & 1.441 & 40 & 1.169 \\
\hline $21 / 12$ & 21 & 8201876529843216 & 1.600 & 42 & 1.223 \\
\hline $22 / 12$ & 22 & 11958560144762940 & 1.796 & 44 & 1.285 \\
\hline $23 / 12$ & 23 & 13434052797314820 & 2.049 & 46 & 1.358 \\
\hline $24 / 12$ & 24 & 11607530825388960 & 2.390 & 48 & 1.447 \\
\hline $25 / 12$ & 25 & 7646075300445120 & 2.877 & 50 & 1.559 \\
\hline $26 / 12$ & 26 & 3770623313530920 & 3.622 & 52 & 1.704 \\
\hline $27 / 12$ & 27 & 1347900319873800 & 4.876 & 54 & 1.902 \\
\hline $28 / 12$ & 28 & 329884270531200 & 7.312 & 56 & 2.189 \\
\hline $29 / 12$ & 29 & 49451047430400 & 13.30 & 58 & 2.635 \\
\hline
\end{tabular}

Table 2: The ratio of the estimated number divided by exact number of labeled 2connected planar graphs with $n$ vertices and $q$ edges

boundaries. The relative errors, though large for such small $n$, seem to drop consistently as $n$ increases (for a fixed value of $q / n$ ) except for the "boundary" case $q / n=13 / 12$. 


\section{References}

[1] E.A. Bender, E.R. Canfield, Z.C. Gao, and L.B. Richmond, Submap density and asymmetry results for two parameter map families, Combinat. Probab. and Comput. 6 (1997) 17-25.

[2] E.A. Bender, Z.C. Gao, and L.B. Richmond, Submaps of maps I: General 0-1 laws, J. Combin. Theory B 55 (1992) 104-117.

[3] E.A. Bender and L.B. Richmond, Central and local limit theorems applied to asymptotic enumeration II: Multivariate generating functions, J. Combin. Theory A 34 (1983) 255-265.

[4] E.A. Bender and L.B. Richmond, The asymptotic enumeration of rooted convex polyhedra, J. Combin. Theory B 3 (1994) 276-283.

[5] R.C. Mullin and P.J. Schellenberg, The enumeration of c-nets via quadrangulations, J. Combin. Theory 4 (1968) 259-276.

[6] A.M. Odlyzko, Asymptotic Enumeration Methods, in "Handbook of Combinatorics", Vol. II (R.L. Graham, M. Grotschel and L. Lovasz Ed), Elsevier Science B.V., 1995.

[7] L.B. Richmond and N.C. Wormald, Almost all maps are asymmetric, J. Combin. Theory B 63 (1995) 1-7.

[8] W.T. Tutte, A census of planar maps, Canad. J. Math. 15 (1963) 249-271.

[9] T.R.S. Walsh, Counting labeled three-connected and homeomorphically irreducible two-connected graphs, J. Combin. Theory B 32 (1982) 1-11.

[10] H. Whitney, Congruent graphs and the connectivity of graphs, Amer. J. Math. 54 (1932) 150-168.

[11] E.M. Wright, The number of unlabeled graphs with many nodes and edges, Bull. of the Amer. Math. Soc., 78, (1972), 1032-1034. 